\title{
Case Report on Obsessive-Compulsive Disorder
}

\author{
Samuel Vanlalpeka ${ }^{1}$, Jaya Gawai ${ }^{2}$, Manoj Patil ${ }^{3}$ \\ ${ }^{1}$ M.Sc. Nursing, ${ }^{2}$ Associate Professor, Dept. of Mental Health Nursing, Smt. Radhikabai Meghe Memorial College \\ of Nursing Sawangi (Meghe), Wardha Datta Meghe Institute of Medical Sciences (Deemed to be University) \\ Maharashtra, India, ${ }^{3}$ Research Consultant, Jawaharlal Nehru Medical College, Datta Meghe Institute of Medical \\ Sciences (DU) Sawangi (M), Wardha India
}

\begin{abstract}
Introduction: Obsessive-compulsive disorder is an anxiety disorder in which people have recurrent, undesirable thoughts, ideas or sensations (obsessions) that make them feel compelled to go and do something (compulsion) repeatedly. Repetitive habits, such as washing hands, inspecting items or bathing, can substantially interfere with the everyday routines and social experiences of a individual.

Symptoms: Behavioral changes like hyperactivity, impulsivity, repetitive movement, perfectionism and social isolation. Mood changes likes anxiety, anger and apprehension. Psychological changes like sleep disturbance, fear, repeatedly going over thoughts and narcissism.

Investigation: $\mathrm{Hb} \%$ - $13 \mathrm{mg} / \mathrm{dl}$; Glucose plasma random - $86 \mathrm{mg} \%$; Serum Globulin- 3.2gm \%; serumProtein- $7 \mathrm{gm} \%$; HIV, HCV, HBsAg, VDRL, all of these tests were negative.

Surgical Management: Patient have no past surgical history.

Medical Management: Patient was treated with Ativan (Lorazepam) 3mg q6hr per oral and Therapies like Aversion therapy and Group Psychotherapy was also used.

Nursing Management: Assess for physical, psychological and social data. Aware for Impact of obsessions and compulsions on physical health, mood, self-esteem and natural capacity to cope. Remember that the defense mechanisms used, the nature or structure of the feeling, the suicidal risk, the capacity to work and the space accessible for social support services.
\end{abstract}

Conclusion: Patient was admitted to AVBRH and was diagnosed to have Obsessive-compulsive disorder. The got appropriate treatment and therapy and his condition has improved well.

Keywords: Obsessive-compulsive disorder, anxiety, compulsion, obsession, lorazepam, narcissism.

\section{Introduction}

Individuals with obsessive-compulsive personality disorder are very serious and Obsessive-compulsive personality disorder is very severe and formal, and has emotional difficulty. They are highly rigid, perfectionist, and keen on law. They are resistant to change about how tasks will be handled, and dedicate themselves to success to the exclusion of personal satisfaction. An extreme fear of making mistakes leads to a challenging decisionmaking process. The disease is relatively widespread and appears more often in men than in women. It appears to be most common in the oldest children within the family group. ${ }^{1}$

The obsessive-compulsive disorder is, according to ICD9, a state in which "the outstanding symptom is a feeling of subjective compulsion which must be resisted - to carry out some action, to dwell on an idea, to recall an experience, or ruminate on an abstract topic. Unwanted thoughts, which include the insistency of words or ideas are perceived by the patient to be inappropriate or nonsensical. The obsessional urge or idea is recognized as alien to the personality, but as coming from within the 
self. Obsessional rituals are designed to relieve anxiety, e.g. washing the hands to deal with contamination. Attempts to dispel the unwelcome thoughts or urges may lead to a severe inner struggle, with intense anxiety." 2

Patient information: A male patient 47 years from Halwadi, Wardha was admitted to Psychiatric Ward, AVBRH on $10^{\text {th }}$ January 2020 with a case of Obsessivecompulsive disorder.

Present Medical History: My patient was apparently asymptomatic 2 years back when he was working in a private company while he was firing from his job due to his lack of responsibility according to his employer. Since then, he started maintaining selfdisciplined and directing himself to his work with fear of loosing the new job again. His behavior getting very excessive that leads to difficulty in coping with family and the society. He was brought to AVBRH by his wife on 10/01/2020, when he was diagnosed with Obsessivecompulsive disorder.

Past Medical History: My patient was apparently asymptomatic 2 years back when he was fired from his job due to lack of responsibility. He started devoting himself to his new job, maintaining new job self-disciplined that eventually leads to excessive behavior disorder like sleep disturbance, lack of interaction, perfectionist, difficulty in completing task due to perfectionism and strict standards, maintaining extreme hygiene of self and surroundings.

Family History: My patient belongs to a nuclear family, middle class living with his wife and two sons in their own house in Halwadi, Wardha. He is working as aa clerk in a private school and is a breadwinner of the family. His two sons, one is $11^{\text {th }}$ standard and the other is $9^{\text {th }}$ grade. His wife has a small grocery store in their house.

Clinical Findings: Behavioral changes like hyperactivity, impulsivity, repetitive movement, perfectionism and social isolation. Mood changes likes anxiety, anger and apprehension. Psychological changes like sleep disturbance, fear, repeatedly going over thoughts and narcissism.

Predisposing Factors: From a psychoanalytical point of view, one of overcontrol is the parental style in which the adult with obsessive-compulsive personality disorder was reared. Such parents want their children to live up to and reject their set standards of behaviour if they do not. Praise is bestowed on the child with far less frequency than punishment for unwanted behaviors. Individuals are specialists in this setting of knowing what they can not do to escape retribution and criticism, rather than what they should do to gain respect and praise. They learn to follow strict rules and regulations. Significant accomplishment are expected, taken for granted and acknowledged only occasionally by their parents, whose responses and decisions are restricted to pointing out transgressions and infringement of the rules. ${ }^{1}$

Pathophysiology: Anxiety, obsessivecompulsive,most likely and associated disorder are caused by multiple factors. A graphic description of this multiple association hypothesis is provided in the Stress/ Adaptation Transactional Model.

Mental Status Examination: A mental status examination was performed on my patient and it was found out that there was an impaired in the stream of thought and in the insight, the score was 2 i.e. the client denies of him having mental illness.

Diagnostic Assessment: $\mathrm{Hb} \%$ - $13 \mathrm{mg} / \mathrm{dl}$; Glucose plasma random - $86 \mathrm{mg} \%$; Serum Globulin- 3.2gm \%; serum-Protein- $7 \mathrm{gm} \%$; HIV, HCV, HBsAg, VDRL, all of these tests were negative.

Therapeutic Intervention: Tab Ativan (Lorazepam) 3mg PO x q6h. Therapies like Aversion therapy and Group Psychotherapy was also used.

\section{Discussion}

A male client of 47 years old from Halwadi was admitted to Psychiatric ward, AVBRH on $10^{\text {th }}$ January 2020 with a complaint of strict personality and disciplined, which cause him to interact or cope with his family or society (according to the patient). According to the patient's wife, the patient was an extreme selfdisciplined, perfectionist, and he also wanted to change the bedsheet, clothes, shoes and blankets every day and he was very violent when they disobey him and this behavior had started in the past 2 years ago. $\mathrm{He}$ was diagnosed as Obsessive-compulsive disorder. As soon as he was admitted to hospital investigations were done and appropriate treatment were started. After getting treatment, he shows great improvement and the treatment was still going on till my last date of care $^{2,3}$.

A study was done on, “An epidemiological study and 
severity assessment of obsessive-compulsive disorder in Warangal region, India". The study aimed at to clinically assess the prevalence and assess the severity of obsessivecompulsive disorder (OCD) in Warangal region, India. A prospective observational study was done for a duration of 6 months i.e. from July-December 2013 at various Neuropsychiatric Centres in Warangal region. A total of 113 patients (male: 65, female: 48) were screened using specially designed data collection form to collect and record demographic data. Structured interviews were conducted to obtain Yale Brown Obsessive- Compulsive Scale (YBOCS) scores. All the data were analysed by ANOVA and Chi Square test using Statistical Package for Social Sciences (SPSS) software version by dividing the patients into age groups of decades from 11 to 60 . It was found out This obsessive-compulsive disorder was more common in subjects between the ages of 21 and $30(46 \%)$ and literate in patients $(98.2 \%)$. More patients were married $(61.6 \%)$ than single patients $(38 \%)$. The most prevalent obsession was fear of harming $(81.25 \%)$ while of the most prevalent compulsions was repeating (76.1\%). Based on YBOC scale, OCD severity was categorized into subclinical (2.7\%), mild (23.9\%), moderate $(41.6 \%)$, severe $(23 \%)$ and extreme $(8.9 \%)$. The study concluded that the prevalence of OCD condition was more among the age group of 21 to 30 years and among literates. Sociodemographic factors like age, socioeconomic status and urban were risk factors in assessing the severity of OCD. ${ }^{3}$ Fewarticles related to various aspects of prevalent psychological problems and associated factors were reviewed ${ }^{4-11}$. Ethical clearanceTaken from institutional ethics committee.

Source of Funding: Self.

\section{Conflict of Interest: Nil.}

\section{References}

1. Townsend M, 'Psychiatric Mental Nursing', Eight edition, Jaypee Publication, 2015; Page 679, 680.537 ,
2. Sreevani R, A Guide to Mental Health and Psychiatric Nursing $4^{\text {th }}$ Edition, Jaypee Brothers Medical Publishers 2016.

3. Sujan Reddy $\mathrm{S}$ et al. An epidemiological study and severity assessment of obsessive-compulsive disorder in Warangal region, India. European Journal of Pharmaceutical and Medical Research. 2016,3(4), 205-210.

4. Gupta, R et al. Clinical Practice Guidelines for Sleep Disorders." Indian Journal of Psychiatry. 2017; 59(5): S116-38.

5. Pal, $\mathrm{S}$ et al. Recognition of Major Depressive Disorder and Its Correlates among Adult Male Patients in Primary Care.Archives of Psychiatry and Psychotherapy. 2018 20(3): 55-62.

6. Patel, T et al. "Prevalence of Alcohol Use Disorders in Hospitalised Male Patients." Archives of Psychiatry and Psychotherapy. 2018 20(4): 47-55.

7. Tripathi, A et al. Gender Differences in ObsessiveCompulsive Disorder: Findings from a Multicentric Study from Northern India.Asian Journal of Psychiatry. 2018 37: 3-9.

8. Gaidhane A, et al. A systematic review on effect of electronic media on diet, exercise, and sexual activity among adolescents. Indian Journal of Community Medicine. 2018;43(5):S56-65.

9. Khatib M, et al. A systematic review on effect of electronic media among children and adolescents on substance abuse. Indian Journal of Community Medicine. 2018;43(5):S66-72.

10. Khatib $\mathrm{M}$ et al. Yoga for improving functional capacity, quality of life and cardiovascular outcomes in people with heart failure. Cochrane Database of Systematic Reviews. 2017(7).

11. Tendolkar, $\mathrm{V}$ et al. Heterogeneous Group Discussion to Improve Reliability and Validity of Data Tool: A Global Mental Health Assessment Tool - Primary Care Version Study." Journal of Datta Meghe Institute of Medical Sciences University. 2017 12(1): 45-50. 